\title{
Structures of individual components of biologically active oxidized lignin material determined by ultra-high resolution mass-spectrometric techniques
}

\author{
Zherebker A. ${ }^{1}$, Kharybin O. ${ }^{1}$, Orlov A. ${ }^{1}$, Lechtenfeld O. ${ }^{2}$, Fedoros E.I. ${ }^{3}$, Perminova I.V. ${ }^{4}$, \\ Nikolaev E.N. \\ ${ }^{1}$ Skolkovo Institute of Science and Technology, Moscow, Russia, a.zherebker@skoltech.ru \\ ${ }^{2}$ Helmholtz-Centre for Environmental Research, Leipzig, Germany \\ ${ }^{3}$ Petrov National Medical Research Center of Oncology, Saint-Petersburg, Russia \\ ${ }^{4}$ Lomonosov Moscow State University, Moscow, Russia
}

doi: 10.36291/HIT.2019.zherebker.052

Oxidized lignin materials possess a wide range of biological activity including hepatoprotection [1]. However, molecular complexity prevents a determination of active species and, consequently, a description of the molecular mechanism of their action. At this regard FTICR MS in combination with isotopic tags provide a unique opportunity to obtain structural information about individual molecular components, which can be used for in silico search of molecular targets. Here, we report on the first combination of several selective labeling techniques to enumerate functional groups and structural fragments of water-soluble product of oxidative degradation of lignin BP-Cx-1.

Carboxylic, carbonyl and phenolic moieties were determined by selective incorporation of deuterium tags via esterification, reduction and acetylation, respectively. In most cases, the numbers of oxygen atoms were covered by these functional groups. Additionally, we observe electrophilic addition of deuterium, which clearly indicates the presence of $\alpha, \beta$-unsaturated carboxyls and carbonyls in the BP-Cx-1 structures. Application of acid-base catalyzed H/D exchange enabled to determine the substitutional pattern of aromatic rings. The obtained results were used to extract structures from the curated database of biological activity ChEMBL, which matched results of FTICR MS analysis and isotopic labeling. The developed approach can be considered as a first step in the understanding of the BP-Cx-1 biological action.

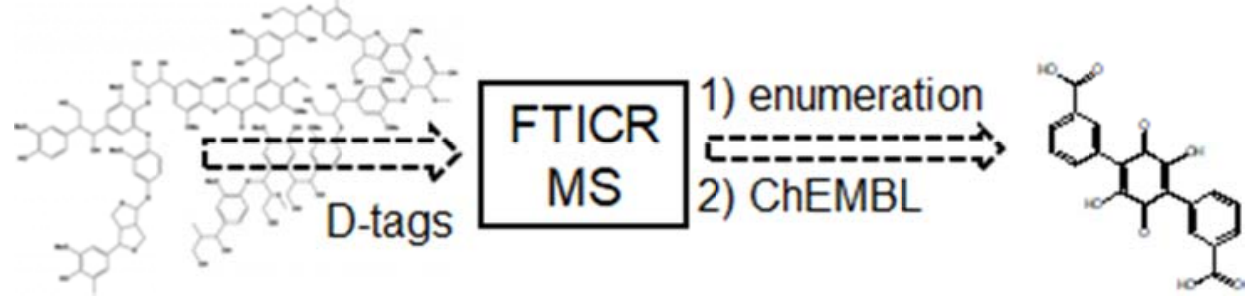

Figure 1. Work-flow for the BP-Cx-1 individual components determination using FTICR $\mathrm{MS}$, isotopic labeling and $\mathrm{ChEMBL}$ data-mining.

Acknowledgements. The work was supported by the Russian Science Foundation (grant no 19-75-0092). The support of IUPAC project 2016-015-2-600 (IP, OL, EN) is kindly appreciated.

References

1. Fedoros E.I. et al. // Oncotarget. 2018. 9:18578-18593. 